\title{
Long-term subjective memory after electroconvulsive therapy
}

Robert Sigström, Axel Nordenskjöld, Anders Juréus, Caitlin Clements, Erik Joas, Erik Pålsson and Mikael Landén

\section{Background}

There have been reports of long-term subjective memory worsening after electroconvulsive therapy (ECT).

\section{Aims \\ To study the prevalence and risk factors of long-term subjective memory worsening among patients receiving ECT in routine clinical practice.}

\section{Method}

Patients ( $n=535$, of whom 277 were included in the final analysis) were recruited from eight Swedish hospitals. Participants' subjective memory impairment was assessed before ECT and a median of 73 days after ECT using the memory item from the Comprehensive Psychopathological Rating Scale. Participants also rated their pre-ECT expectations and post-ECT evaluations of the effect of ECT on memory on a 7-point scale. We used ordinal regression to identify variables associated with subjective memory worsening and negative evaluations of the effect of ECT on memory.

\section{Results}

Comparisons of pre- and post-ECT assessments showed that subjective memory worsened in $16.2 \%$ of participants, remained unchanged in $52.3 \%$ and improved in $31.4 \%$. By contrast, when asked to evaluate the effect of ECT on memory after treatment $54.6 \%$ reported a negative effect. Subjective memory worsening was associated with negative expectations before ECT, younger age and shorter duration of follow-up.

\section{Conclusions}

Although subjective memory improved more often than it worsened when assessed before and after ECT, a majority of patients reported that ECT had negative effects on their memory when retrospectively asked how ECT had affected it. This might suggest that some patients attribute pre-existing subjective memory impairment to ECT. Clinicians should be aware that negative expectations are associated with subjective worsening of memory after ECT.

\section{Declaration of interest}

A.J. is currently employed at the Swedish Medical Products Agency, Uppsala, Sweden; the views expressed in this paper are the personal views of the author and not necessarily the views of the government agency. M.L. declares that, over the past 36 months, he has received lecture honoraria from Lundbeck Pharmaceutical.

\section{Keywords}

Electroconvulsive therapy; memory; adverse effects; amnesia; cognition.

\section{Copyright and usage}

(c) The Author(s) 2020. This is an Open Access article, distributed under the terms of the Creative Commons Attribution-

NonCommercial-NoDerivatives licence (http://creativecommons. org/licenses/by-nc-nd/4.0/), which permits non-commercial reuse, distribution, and reproduction in any medium, provided the original work is unaltered and is properly cited. The written permission of Cambridge University Press must be obtained for commercial re-use or in order to create a derivative work.
Electroconvulsive therapy (ECT) is an effective and rapid treatment for depression ${ }^{1}$ and selected other psychiatric conditions. ${ }^{2}$ However, the use of ECT has declined in some countries, including the UK. ${ }^{3}$ One explanation might be concerns about negative effects on memory. Although it is generally accepted that memory impairment might occur during and immediately after the treatment, ${ }^{4}$ the question of whether ECT might have long-term effects on memory remains controversial. ${ }^{5,6}$ On the one hand, long-term follow-up studies using standard neuropsychological tests have found that cognition returns to baseline levels, or improves, after ECT. ${ }^{4,7-10}$ On the other hand, case reports ${ }^{11,12}$ and retrospective evaluations of ECT's effect on memory ${ }^{13,14}$ suggest that some people experience long-term subjective memory impairment after ECT. In line with this, subjective memory impairment following ECT correlates poorly with objectively assessed memory function, but strongly with concurrent depressive symptoms. ${ }^{5,8,10}$ A frequently cited review of studies published between 1980 and 2000 estimated that 29-55\% of people experienced negative effects on memory after ECT. ${ }^{15}$ However, these studies may be outdated owing to changes in ECT techniques and have also been criticised for methodological shortcomings such as inability to separate acute and persistent memory effects of ECT. ${ }^{16}$ Thus, there is a need to clarify the magnitude of the problem and its risk factors in current ECT practice.
The aims of this study were to investigate (a) the prevalence of and risk factors for long-term subjective worsening of memory following ECT and (b) the extent to which results depend on how questions regarding memory are framed to patients.

\section{Method}

\section{Inclusion and exclusion criteria}

The present study is part of the Predictors for ECT (PREFECT) project. Eight Swedish hospitals enrolled study participants between May 2014 and August 2016. Patients $\geq 18$ years of age who were scheduled to receive at least six ECT sessions were eligible for inclusion regardless of clinical diagnosis. In total, 535 individuals consented to participate in the study. Of these, four individuals were included twice, but data were used from only one of these occasions.

Figure 1 presents a flowchart of included and excluded participants. Participants were excluded if they had no data in the Swedish National Quality Register for ECT (Q-ECT), received fewer than three ECT sessions, were interviewed for follow-up less than 16 days after the last ECT treatment (the lower bound for long-term cognitive effects of ECT as suggested by others ${ }^{4}$ ) or failed to respond to the Comprehensive Psychopathological Rating Scale (CPRS) subjective memory item pre- or post-ECT. Forty-eight 
11 No data in National Quality Register for ECT

4 Received fewer than three ECT sessions

14 Follow-up shorter than 16 days

68 No pre-ECT data on subjective memory impairment

161 No post-ECT data on subjective memory impairment

277 Included in the present study

Fig. 1 Flowchart of included and excluded participants

participants were unable to respond to the baseline CPRS memory item owing to their clinical status and 46 participants refused further contact after the baseline interview. For the remaining participants who failed to respond to the memory question $(n=135)$, the reason was not specified. In total, 277 participants (51.8\%) were included in the present study. Table 1 presents a comparison of included and excluded participants.

We used the Q-ECT to track additional ECT sessions (new series or maintenance treatment) between the end of the index series and the follow-up interview. The Q-ECT, launched in 2011, covered $89 \%$ of all ECT series carried out in Sweden in $2014 .{ }^{17}$ A follow-up telephone interview was completed a median of 73 days (interquartile range $\mathrm{IQR}=52-101$ days) after the last ECT session recorded in the Q-ECT.

\section{ECT procedure}

Electroconvulsive therapy was administered using bidirectional, constant-current, brief-pulse devices from Mecta (Mecta Corp, Lake Oswego, Oregon, USA) or Thymatron (Somatics Inc., Lake Buff, Illinois, USA). For anaesthesia, propofol or thiopental was used. Suxamethonium was used for muscle relaxation. Seizure duration was registered with electroencephalography. From the Q-ECT, we retrieved data on electrode placement (right unilateral according to d'Elia versus bilateral at the first or last session of each treatment series), charge $(\mathrm{mC})$ at the first session and pulse width at the first session (dichotomised into ultrabrief (0.25-0.47 $\mathrm{ms}$ ) versus brief (0.5-1.0 ms)). All participating clinics administered ECT three times a week: Monday, Wednesday and Friday.

\section{Assessment of subjective memory impairment}

Subjective memory impairment was assessed using the Comprehensive Psychopathological Rating Scale (CPRS) ${ }^{18}$ memory item during an in-person interview pre-ECT and by telephone post-ECT. The CPRS is a semi-structured interview covering a wide range of psychiatric symptoms and signs. It was specifically designed to have high sensitivity to change and it has good reliability. ${ }^{19}$ Several psychometric instruments, such as the well-known MontgomeryÅsberg Depression Rating Scale (MADRS), have been constructed using selected items from the CPRS. The memory item assesses current subjective memory impairment compared with previous ability. Interviewers rated respondents' answers to the CPRS memory item on a scale from $0-6$. Ratings $0-1$ indicate no memory impairment, 2-3 indicate occasional lapses of memory, 4-5 indicate disturbing memory impairment, and 6 indicates complete inability to remember. Thus, ratings 4-6 indicate symptoms that are clinically significant, whereas ratings 2-3 indicate discernible symptoms that may or may not be clinically significant. We labelled ratings $4-6$ as clinically significant and $2-3$ as transient memory impairment.

In line with previous research using the CPRS in this context, ${ }^{20}$ we defined subjective memory worsening as the post-ECT CPRS rating being $\geq 2$ points worse than the pre-ECT rating. Since this definition includes changes that may be of small clinical significance, for example a change from no impairment (rating 0) pre-ECT to occasional lapses of memory (a rating of 2) post-ECT, we also calculated the proportion of participants who had both $\geq 2$ points worsening on the post-ECT CPRS rating and a post-ECT rating $\geq 4$ points (i.e., clinically significant memory impairment). Subjective memory improvement was defined as $\geq 2$ points improvement, whereas participants with $<2$ points change in either direction were considered unchanged with respect to memory.

\section{Participants' expectation and evaluation of the effect of ECT on memory}

Participants also completed the Global Self-Evaluation of Memory and of Mood (GSE-My and GSE-Md). ${ }^{14}$ Before ECT, these instruments included questions about what effect the participants expected ECT to have on memory and mood. Answers were rated on a Likert scale ranging from 1 (extremely negative) to 7 (extremely positive). After ECT, the same questions were used to capture participants' retrospective evaluation of ECT's effect on memory. 
Table 1 Characteristics of included and excluded participants

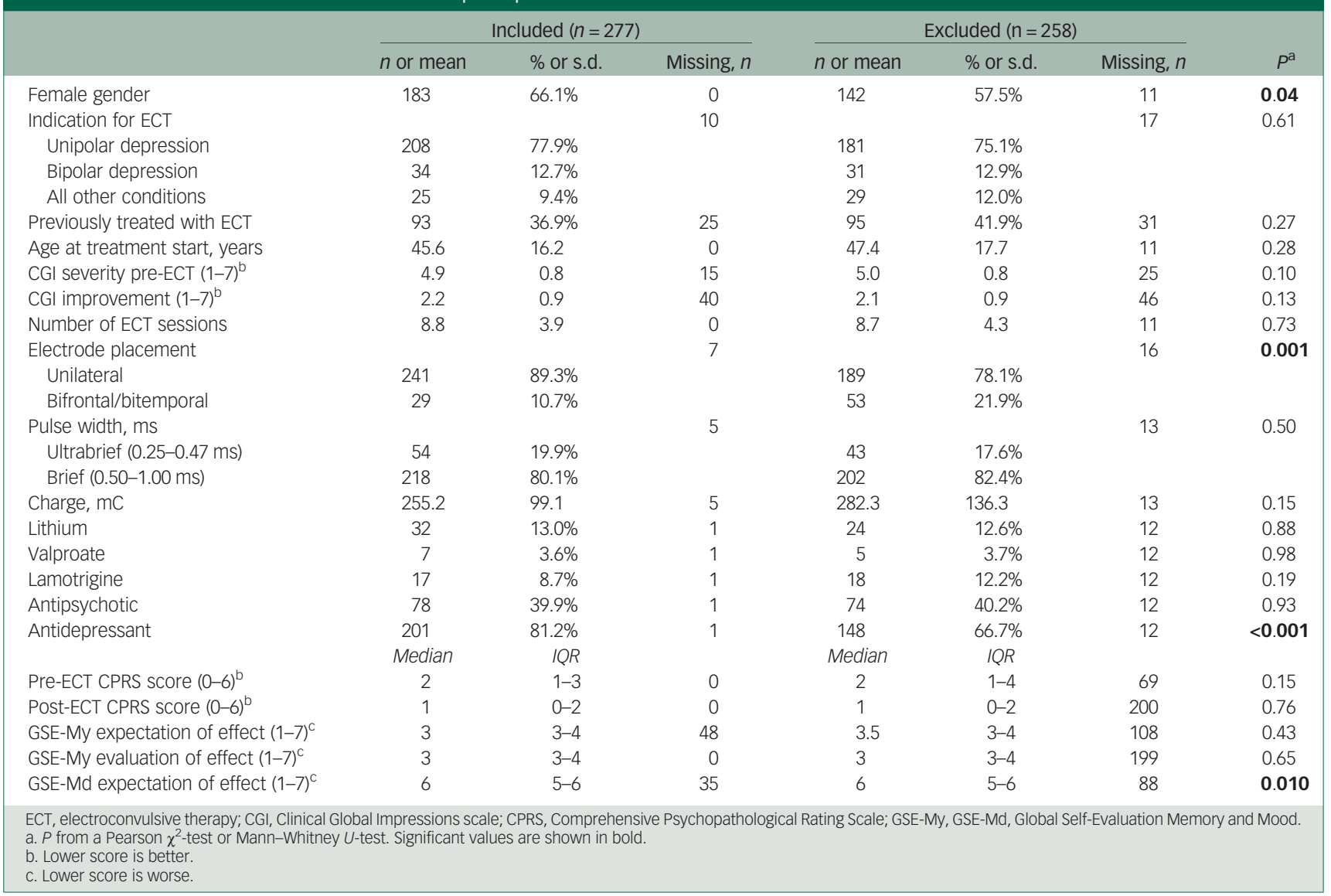

\section{Other variables}

Before ECT, we recorded use of lithium, valproate, lamotrigine, antidepressants and antipsychotics. Other variables were retrieved from the Q-ECT: age, gender, indication for ECT (unipolar depression, ICD-10 codes F32.0-F32.9; bipolar depression, F31.3-F31.5; and 'other', including all other indications), previous treatment with ECT (yes/no), pre-ECT Clinical Global Impressions (CGI) severity scale and post-ECT CGI improvement scale, ${ }^{21}$ the number of ECT sessions between baseline and follow-up, and the time elapsed between the last ECT session and follow-up. Missing data for each variable are displayed in Table 1.

\section{Ethics}

The authors assert that all procedures contributing to this work comply with the ethical standards of the relevant national and institutional committees on human experimentation and with the Helsinki Declaration of 1975, as revised in 2008. All procedures involving patients were approved by the Regional Ethical Review Board in Stockholm, Sweden (approval no. 2012/1969-31/1). Written informed consent was obtained from all participants.

\section{Statistical analysis}

We tested differences in categorical and continuous variables using Pearson $\chi^{2}$ - and Mann-Whitney $U$-tests respectively. We used Wilcoxon signed-rank tests to test changes in CPRS and GSE-My scores between pre- and post-ECT.

We used ordinal regression to analyse risk factors for two outcomes: (a) change in CPRS rating from pre- to post-ECT and (b) negative retrospective evaluations of the memory effects of ECT. Since there was a substantial amount of missing data, we imputed data on covariates using multivariate imputation by chained equations (MICE). For the first outcome, we used level2 predictive mean matching (PMM) as the data were transformed for longitudinal analysis. However, all imputed variables were constant across time for each person. ${ }^{22}$ For the second outcome, we used PMM to impute missing values. We created 50 imputed data-sets for each analysis described below and conducted pooled analyses.

For analysis of change in the CPRS variable, we used an ordinal mixed-effects model. ${ }^{23}$ Owing to the sparse number of responses for some alternatives we collapsed the dependent variables (CPRS ratings pre- and post ECT) into three-level variables (0-1, 2-3, 4-6). For each covariate, we created a separate model including the main effect of the covariate, the main effect of time and the interaction between covariate and time. When examining the retrospective evaluation of ECT's effect on memory (GSE-My), we similarly collapsed the dependent variable into three classes $(1-2,3,4-7)$ from the original seven. We then used ordinal regression to analyse the effect of each covariate on the dependent variable. Lastly, we constructed multivariate models for both outcomes in three steps by (a) including all variables with $P<0.25$ in univariate models, (b) backward selection by removing the variable with the highest $P$-value in each step until only significant effects remained and (c) comparing point estimates of all variables in this reduced model with those in the full model in step (a) to investigate whether we had missed any important confounders. If there was no substantial difference in point estimates, we used the reduced model as our final model.

Results were considered statistically significant when $P<0.05$ (two-tailed). Analyses were carried out using IBM SPSS Statistics v.24 for Windows and R v.3.6.0 for Windows. 


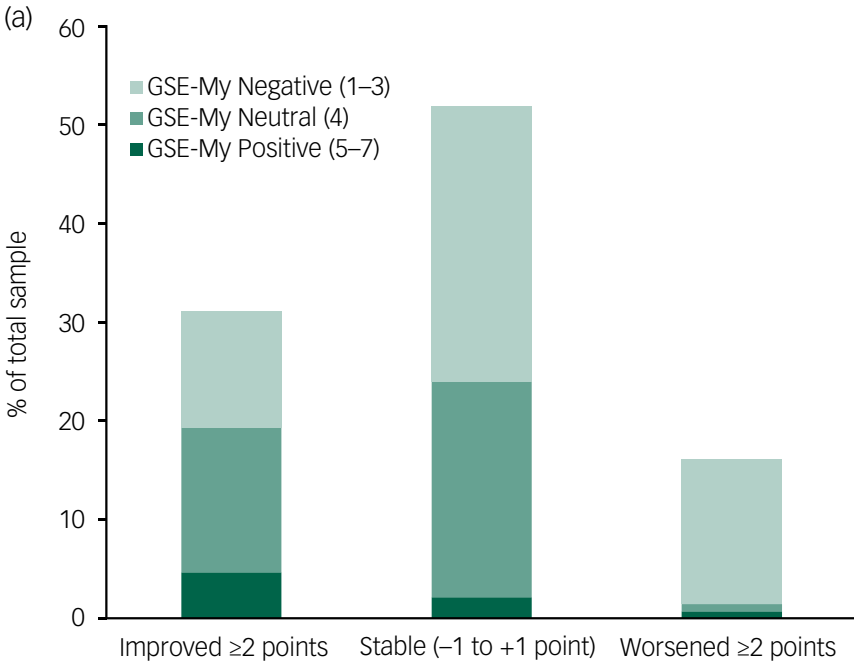

CPRS

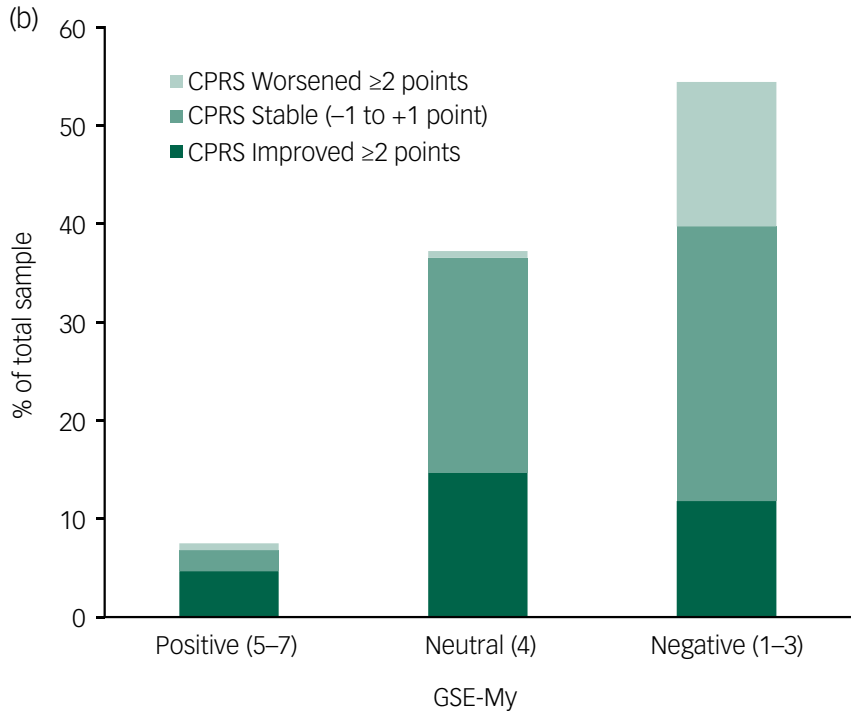

Fig. 2 Subjective memory variables pre- and post-electroconvulsive therapy. (a) Subjective memory change from pre- to post-ECT according to Comprehensive Psychopathological Rating Scale (CPRS) score. (b) Post-ECT evaluation of ECT effect on memory according to Global SelfEvaluation Memory (GSE-My) score. Total sample: $n=277$; for exact numbers and percentages, see the text and supplementary Table 2 .

\section{Results}

Table 1 shows characteristics of the included and excluded participants. Included participants were more likely to be female, to be taking antidepressant medication, to receive unilateral ECT and to have more positive expectations about the mood effects of ECT.

\section{Subjective memory change from pre- to post-ECT}

Overall, subjective memory as assessed using the CPRS improved from pre-ECT assessment (median 2, IQR $=1-3$ ) to post-ECT assessment (median $1, \mathrm{IQR}=0-2$, Wilcoxon signed-rank test $Z=$ $-4.39, P<0.001)$. Memory was unchanged in $52.3 \%(n=145)$ and improved in $31.4 \%(n=87)$ of participants. Subjective memory worsening occurred in $16.2 \%(n=45)$, as seen in Fig. 2 and supplementary Table 1 (available at https://doi.org/10.1192/bjo.2020.9). Among those with subjective memory worsening, 22 participants (7.9\% of the total sample) also reported clinically significant memory impairment post-ECT.

\section{Expectations and evaluations of ECT effect on memory}

The expectations of ECT's effect on memory before treatment were less negative (median $3, \mathrm{IQR}=3-4$ ) than the evaluations after treatment (median 3, IQR $=3-4, Z=-5.85, P<0.001$ ). Before ECT, $6.6 \%(n=15)$ had very negative expectations (GSE-My rating $1-2)$ regarding what effect ECT would have on their memory. After ECT, $23.1 \%(n=64)$ considered ECT to have had a very negative effect on their memory (supplementary Table 1).

\section{Relationship between memory change and post-ECT evaluations}

The GSE-My evaluations of memory effects after treatment were more negative than the change in subjective memory as assessed with the CPRS (Fig. 2 and supplementary Table 2). For example, most of those with retrospective negative evaluations after treatment did not have memory worsening according to the CPRS assessment.

\section{Variables associated with subjective memory worsening}

Table 2 presents the time interaction effects of all covariates on the CPRS memory item. In the final multivariate model after multiple imputation, increasing age (odds ratio (OR) per 10-year increase $0.78,95 \%$ CI $0.63-0.97$ ) and longer time to follow-up (OR per 4 additional weeks $0.68,95 \%$ CI $0.51-0.91$ ) were associated with less subjective memory worsening. Negative expectations before treatment were associated with more subjective memory worsening (OR per 1-point more negative evaluation 1.83, 95\% CI 1.28-2.61). Analyses without imputation resulted in similar estimates (supplementary Table 4). Main effects for all variables are displayed in supplementary Table 3. More negative pre-ECT expectations of memory effects were associated with less baseline subjective memory impairment (OR per 1-point more negative expectation $0.73,95 \%$ CI $0.56-0.94)$.

Table 3 presents associations between a more negative retrospective evaluation of ECT's memory effects and all covariates. In the final multivariate model after multiple imputation, increasing age (OR per 10 -year increase $0.73,95 \%$ CI $0.63-0.85$ ) reduced the risk of negative evaluation. Subjective memory impairment preECT (OR per 1-point worse evaluation 1.31, 95\% CI 1.11-1.55), pre-ECT expectations (OR per 1-point worse evaluation 1.30, 95\% CI $1.03-1.66)$ and valproate use $(\mathrm{OR}=4.00,95 \%$ CI $1.07-14.93)$ were associated with higher risk for a negative evaluation. Analyses without imputation resulted in similar estimates (supplementary Table 5).

\section{Discussion}

We studied long-term effects of ECT on subjective memory in routine clinical practice. We not only used the CPRS memory item to assess memory before and after treatment, but also asked participants before treatment what effect they expected ECT to have on their memory. After treatment, we asked them to evaluate the effect ECT had had on their memory. For a clear majority of participants, subjective memory improved or remained unchanged from pre- to post-ECT according to the CPRS assessment, but it 


\begin{tabular}{|c|c|c|c|c|c|c|}
\hline & \multicolumn{3}{|c|}{ Univariate model } & \multicolumn{3}{|c|}{ Final multivariate model } \\
\hline & OR & $95 \% \mathrm{Cl}$ & $P$ & $\mathrm{OR}$ & $95 \% \mathrm{Cl}$ & $P$ \\
\hline $\begin{array}{l}\text { Female gender } \\
\text { Indication for } \mathrm{ECT}\end{array}$ & \multicolumn{5}{|c|}{ Indication for ECT } & \\
\hline Unipolar depression & Refe & & & & & \\
\hline Bipolar depression & 1.25 & $0.46-3.39$ & 0.66 & & & \\
\hline All other conditions & 1.99 & $0.58-6.85$ & 0.26 & & & \\
\hline Previously treated with ECT & 1.48 & $0.73-2.99$ & 0.28 & & & \\
\hline Lithium & 1.38 & $0.52-3.67$ & 0.52 & & & \\
\hline Valproate & 2.53 & $0.43-15.08$ & 0.31 & & & \\
\hline Lamotrigine & 2.64 & $0.83-8.38$ & 0.10 & & & \\
\hline Antipsychotic & 1.05 & $0.53-2.09$ & 0.89 & & & \\
\hline Antidepressant & 0.78 & $0.34-1.81$ & 0.57 & & & \\
\hline Bifrontal/bitemporal ECT (v. unilateral) & 0.94 & $0.30-2.94$ & 0.92 & & & \\
\hline Brief pulse width (v. ultrabrief) & 1.84 & $0.79-4.28$ & 0.16 & & & \\
\hline Age, per 10 years & 0.75 & $0.60-0.93$ & 0.01 & 0.78 & $0.63-0.97$ & 0.03 \\
\hline Duration of follow-up, per 4 weeks & 0.71 & $0.54-0.94$ & 0.02 & 0.68 & $0.51-0.91$ & 0.01 \\
\hline Number of ECT sessions, per session & 1.11 & $1.02-1.20$ & 0.02 & & & \\
\hline Charge, per $100 \mathrm{mC}$ & 0.85 & $0.61-1.19$ & 0.35 & & & \\
\hline CGI severity pre-ECT $(1-7),{ }^{\mathrm{b}}$ per point increase & 0.70 & $0.44-1.10$ & 0.11 & & & \\
\hline CGI improvement $(1-7),{ }^{\mathrm{b}}$ per point increase & 1.67 & $1.13-2.46$ & 0.01 & & & \\
\hline $\begin{array}{l}\text { More negative expectation of memory effect (GSE-My, } \\
1-7 \text { ), per point worsening }\end{array}$ & 1.90 & $1.33-2.70$ & $<0.001$ & 1.83 & $1.28-2.61$ & $<0.001$ \\
\hline $\begin{array}{l}\text { More negative expectation of mood effect (GSE-Md, } \\
\text { 1-7), per point worsening }\end{array}$ & 1.21 & $0.77-1.90$ & 0.40 & & & \\
\hline
\end{tabular}

worsened in $16 \%$ of participants. Despite this, when asked to retrospectively evaluate the effect of ECT on memory, as many as 55\% of participants reported that ECT had had a negative effect on their memory. This demonstrates that the method used to capture subjective memory impairment after ECT has a large influence on the results. Factors that were significantly associated with worsening of memory as assessed using the CPRS were younger age, shorter follow-up time and negative expectations before treatment.

\section{Comparison with previous studies}

Previous reports on the proportion of people who experience longterm negative effects on subjective memory after ECT vary widely (29-55\%). ${ }^{15}$ This study provides a possible explanation for this. The prevalence of negative subjective effects of ECT on memory in our study may be said to be as high as 55\% (retrospective patient evaluation of the impact of ECT on their memory) or as

\section{Table 3 Variables associated with a more negative retrospective evaluation of the effect of electroconvulsive therapy (ECT) on memory}

\begin{tabular}{|c|c|c|c|c|c|c|}
\hline & \multicolumn{3}{|c|}{ Univariate model } & \multicolumn{3}{|c|}{ Final multivariate model } \\
\hline & OR & $95 \% \mathrm{Cl}$ & $P$ & OR & $95 \% \mathrm{Cl}$ & $P$ \\
\hline Female gender & 1.71 & $1.07-2.75$ & 0.03 & 1.69 & $1.03-2.78$ & 0.04 \\
\hline \multicolumn{7}{|l|}{ Indication for ECT } \\
\hline Unipolar depression & \multicolumn{3}{|c|}{ Reference } & & & \\
\hline Bipolar depression & 1.50 & $0.76-2.98$ & 0.24 & & & \\
\hline All other conditions & 0.57 & $0.26-1.29$ & 0.18 & & & \\
\hline Previously treated with ECT & 1.03 & $0.65-1.64$ & 0.90 & & & \\
\hline Lithium & 1.61 & $0.84-3.05$ & 0.15 & & & \\
\hline Valproate & 3.88 & $1.04-14.44$ & 0.04 & 4.00 & $1.07-14.93$ & 0.040 \\
\hline Lamotrigine & 0.91 & $0.42-1.97$ & 0.80 & & & \\
\hline Antipsychotic & 1.03 & $0.66-1.60$ & 0.91 & & & \\
\hline Antidepressant & 1.06 & $0.60-1.86$ & 0.85 & & & \\
\hline Bifrontal/bitemporal ECT (v. unilateral) & 0.69 & $0.34-1.42$ & 0.32 & & & \\
\hline Brief pulse width ( $v$. ultrabrief) & 1.04 & $0.60-1.79$ & 0.90 & & & \\
\hline Age, per 10 years & 0.74 & $0.64-0.85$ & $<0.001$ & 0.73 & $0.63-0.85$ & $<0.001$ \\
\hline Duration of follow-up, per 4 weeks & 1.04 & $0.87-1.24$ & 0.68 & & & \\
\hline Number of ECT sessions, per session & 1.04 & $0.99-1.10$ & 0.14 & & & \\
\hline Charge, per $100 \mathrm{mC}$ & 0.93 & $0.74-1.16$ & 0.50 & & & \\
\hline CGI severity pre-ECT (1-7), ${ }^{\mathrm{b}}$ per point increase & 0.91 & $0.68-1.22$ & 0.54 & & & \\
\hline More subjective memory impairment pre-ECT $(C P R S, 0-6),{ }^{b}$ per point increase & 1.20 & $1.03-1.40$ & 0.02 & 1.31 & $1.11-1.55$ & 0.002 \\
\hline CGI improvement $(1-7),{ }^{b}$ per point increase & 1.25 & $0.96-1.62$ & 0.10 & & & \\
\hline More negative expectation of memory effect (GSE-My, 1-7), per point worsening & 1.29 & $1.04-1.60$ & 0.02 & 1.30 & $1.03-1.66$ & 0.029 \\
\hline More negative expectation of mood effect (GSE-Md, 1-7), per point worsening & 1.18 & $0.88-1.60$ & 0.27 & & & \\
\hline
\end{tabular}


low as $8 \%$ ( $\geq 2$ points worsening on the CPRS memory item in conjunction with clinically significant subjective memory impairment post-ECT). The highest estimate of subjective memory worsening following ECT in this study is on a par with the highest estimate previously reported, ${ }^{24}$ which interestingly was based on similar retrospective questions. The GSE-My has rarely been used in previous ECT research, but the expectations and evaluations of ECT's effect on memory in the present study are similar to a previous naturalistic study that used the GSE-My in an American setting. ${ }^{14}$ By contrast, we found that subjective memory worsened in only $16 \%$ when assessed using the CPRS before and a median of 73 days after ECT. This is lower than in a study that used the same instrument to assess memory worsening immediately after ECT $(25 \%),{ }^{20}$ suggesting that subjective memory impairment subsides with time. Indeed, we found that shorter follow-up time was associated with more subjective memory worsening. These results also align with studies of objectively assessed cognitive function, where impaired memory immediately following ECT returned to baseline levels or improved at long-term follow-up. ${ }^{4}$

Previous studies have also found discrepancies when comparing retrospective global patient evaluations administered post-ECT and score change on subjective memory questionnaires administered pre- and post-ECT. ${ }^{13,14}$ Some have attributed the discrepancy to the difference in the type of memory assessed: global evaluations cover all types of memory impairment, including retrograde amnesia, whereas structured questionnaires only cover certain aspects of memory. However, we found similar discrepancies despite comparing a global patient evaluation with a global memory assessment administered pre- and post-ECT. We therefore propose that retrospective evaluations yield a more negative picture than a comparison of pre- and post-ECT assessments.

\section{Validity of the study method}

Given the subjective nature of the phenomenon under study, it has been argued that taking pre-ECT subjective memory into account is not a valid approach. ${ }^{12}$ However, the validity of retrospective evaluations as measures of long-term side-effects of ECT can also be questioned. First, amnesia for the period surrounding the treatment challenges patients' ability to evaluate change in memory from preto post-ECT. Second, a retrospective evaluation implicitly suggests a causal explanation ('How has ECT affected your memory?'), which is virtually impossible for the individual patient to appraise among the myriad of factors that might affect memory function. Third, negative retrospective evaluations may refer to the well-known phenomenon of retrograde and anterograde amnesia for the treatment period, rather than persistent, disabling effects on memory function. ${ }^{24,25}$ Finally, we found that subjective memory impairment before ECT was associated with a more negative evaluation of ECT's memory effect. This provides empirical support for the notion that negative evaluations of ECT's memory effects may in some cases be due to patients attributing pre-existing subjective memory impairment to ECT. Speculatively, such beliefs might be modifiable by interventions during and after the treatment period to mitigate negative memory effects of ECT.

\section{Participants' characteristics associated with subjective memory impairment}

In our study, more negative expectations of ECT's memory effects were consistently associated with both subjective memory worsening following ECT and a negative retrospective evaluation. Interestingly, participants' expectations of the mood effects of ECT were not associated with negative effects on memory. It is therefore unlikely that the association between more negative expectations and subjective memory impairment was a result of generally negative attitudes towards ECT. Instead, it might reflect a specific nocebo effect of negative expectations or a placebo effect of positive expectations.

Similar to previous studies, ${ }^{13,14,20}$ we found younger age to be associated with a worse subjective memory outcome after ECT according to both outcome measures. This contrasts with studies of long-term objective memory effects of ECT, which have not found a clear relationship with age. ${ }^{4}$ In the present study, no other factor confounded or mediated this association. A similar finding was made in a previous study of people with depression not treated with ECT, in which younger age was associated with having more subjective cognitive impairment relative to objective cognitive function. ${ }^{26}$ Potential explanations for this higher sensitivity to cognitive disturbance among younger people include more exposure to cognitively demanding and distracting activities.

We observed no effect of bilateral ECT or pulse width, which have been found to influence objective cognitive impairment after ECT. ${ }^{13,27}$ However, our observational study is limited by possible confounding by indication. Also, bilateral ECT was used in few participants $(11 \%)$ and the variation in pulse width was small with a relatively short maximum pulse width $(1 \mathrm{~ms})$. Regarding medications, we found that valproate was associated with a more negative evaluation of ECT's memory effects. This finding should be interpreted with caution given the small number of participants taking valproate $(n=7)$. Also, we are not aware of any previous reports linking valproate treatment to adverse cognitive effects of ECT.

\section{Strengths and limitations}

The main strengths of this study are the multicentre design and relatively large, well-characterised sample. The main limitation concerns missing data, which resulted in exclusion of almost half of the original sample from analysis. Thus, we cannot exclude possible bias with respect to subjective memory effects of ECT. Another limitation is that we could not compare the results of our global assessments of subjective memory with objective memory tests or more detailed assessments of subjective memory.

\section{Clinical implications}

The estimated prevalence of subjective memory impairment after ECT is highly dependent on how it is assessed and operationalised. Our findings also suggest that some patients might retrospectively attribute pre-existing subjective memory impairment to ECT. Potential clinical implications of our findings include that the indication for ECT should be carefully considered when patients, especially young individuals, have negative expectations regarding memory effects. Speculatively, negative expectations and attribution of pre-existing memory impairment to ECT may be modifiable by interventions, which might have the potential to reduce the occurrence of subjective memory worsening following ECT.

Robert Sigström $(D, M D, P h D$, Department of Psychiatry and Neurochemistry, Institute of Neuroscience and Physiology, Sahlgrenska Academy, University of Gothenburg, Sweden; Axel Nordenskjöld, MD, PhD, Faculty of Medicine and Health, University Sweden; Axel Nordenskjöld, MD, PhD, Faculty of Medicine and Health, University
Health Care Research Center, Örebro University, Sweden; Anders Juréus, PhD, Department of Medical Epidemiology and Biostatistics, Karolinska Institutet, Sweden; Caitlin Clements, MA, Department of Psychology, University of Pennsylvania, USA; Erik Joas (D), MSC, Department of Psychiatry and Neurochemistry, Institute of Neuroscience and Physiology, Sahlgrenska Academy, University of Gothenburg, Sweden; Erik Pålsson, PhD, Department of Psychiatry and Neurochemistry, Institute of Neuroscience and Physiology, Sahlgrenska Academy, University of Gothenburg, Sweden: Mikael Landén, MD, PhD, Department of Psychiatry and Neurochemistry, Institute of Neuroscience and Physiology, Sahlgrenska Academy, University of Gothenburg; and Department of Medical Epidemiology and Biostatistics, Karolinska Institutet, Sweden

Correspondence: Robert Sigström. Email: robert.sigstrom@gu.se

First received 16 Oct 2019, final revision 10 Feb 2020, accepted 11 Feb 2020 


\section{Supplementary material}

Supplementary material is available online at https://doi.org/10.1192/bjo.2020.9.

\section{Funding}

This research was supported by grants from the Swedish Research Council (2018-02653), the Swedish Foundation for Strategic Research (KF10-0039) and the Swedish Government under the LUA/ALF agreement (ALFGBG-716801).

\section{Acknowledgements}

We thank the participants who contributed to this research; the ECT teams that recruited study participants; the research nurses Martina Wennberg, Birgitta Ohlander, Milka Krestelica and Radja Dawoud, who conducted interviews: the data managers Bozenna Illiadou and Mathias Kardell; and the Swedish National Quality Register for ECT (Q-ECT) for sharing data.

\section{Data availability}

The authors had full and ongoing access to the original data presented and analysed in this study. Due to Swedish legal restrictions, register data cannot be shared. However, the data that support the findings of this study are available from the corresponding author upon reasonable request.

\section{Author contributions}

R.S.: study design, analysis and interpretation of data, drafting of the manuscript. A.N. and A.J.: data acquisition, interpretation of data, revision of the manuscript for important intellectual content. C.C.: interpretation of data, revision of the manuscript for important intellectual content. E.J. data analysis and interpretation, revision of the manuscript for important intellectual content. E.P.: interpretation of data, revision of the manuscript for important intellectual content. M.L.: conception of the study, study design, data acquisition, interpretation of data, revision of the manuscript for important intellectual content.

\section{References}

1 UK ECT Review Group. Efficacy and safety of electroconvulsive therapy in depressive disorders: a systematic review and meta-analysis. Lancet 2003; 361: 799-808

2 Waite J, Easton A (eds). The ECT Handbook (3rd edn) (College Report CR176). RCPsych Publications, 2013.

3 Bickerton D, Worrall A, Chaplin R. Trends in the administration of electroconvulsive therapy in England. Psychiatr Bull 2009; 33: 61-3.

4 Semkovska M, McLoughlin DM. Objective cognitive performance associated with electroconvulsive therapy for depression: a systematic review and meta-analysis. Biol Psychiatry 2010; 68: 568-77.

5 Fraser LM, O'Carroll RE, Ebmeier KP. The effect of electroconvulsive therapy on autobiographical memory: a systematic review. J ECT 2008; 24: 10-7.

6 Semkovska M, McLoughlin DM. Measuring retrograde autobiographical amnesia following electroconvulsive therapy: historical perspective and current issues. J ECT 2013; 29: 127-33.

7 Mohn C, Rund BR. Maintained improvement of neurocognitive function in major depressive disorders 6 months after ECT. Front Psychiatry 2016; 7: 200.

8 Fernie G, Bennett DM, Currie J, Perrin JS, Reid IC. Detecting objective and subjective cognitive effects of electroconvulsive therapy: intensity, duration and test utility in a large clinical sample. Psychol Med 2014; 44: 2985-94.
9 Sackeim HA, Prudic J, Fuller R, Keilp J, Lavori PW, Olfson M. The cognitive effects of electroconvulsive therapy in community settings. Neuropsychopharmacology 2007; 32: 244.

10 Kirov GG, Owen L, Ballard H, Leighton A, Hannigan K, Llewellyn D, et al. Evaluation of cumulative cognitive deficits from electroconvulsive therapy. $\mathrm{Br}$ J Psychiatry 2016; 208: 266-70.

11 Donahue AB. Electroconvulsive therapy and memory loss: a personal journey. J ECT 2000; 16: 133-43

12 Vamos M. The cognitive side effects of modern ECT: patient experience or objective measurement? J ECT 2008; 24: 18-24.

13 Brakemeier EL, Berman R, Prudic J, Zwillenberg K, Sackeim HA. Self-evaluation of the cognitive effects of electroconvulsive therapy. J ECT 2011; 27: 59-66.

14 Berman RM, Prudic J, Brakemeier E-L, Olfson M, Sackeim HA. Subjective evaluation of the therapeutic and cognitive effects of electroconvulsive therapy. Brain Stimul 2008; 1: 16-26.

15 Rose D, Fleischmann P, Wykes T, Leese M, Bindman J. Patients' perspectives on electroconvulsive therapy: systematic review. BMJ 2003; 326: 1363.

16 Bergsholm P. Patients' perspectives on electroconvulsive therapy: a reevaluation of the review by Rose et al on memory loss after electroconvulsive therapy. J ECT 2012; 28: 27-30.

17 Nordanskog P, Hulten M, Landen M, Lundberg J, von Knorring L, Nordenskjold A. Electroconvulsive therapy in Sweden 2013: data from the national quality register for ECT. J ECT 2015; 31: 263-7.

18 Åsberg M, Montgomery SA, Perris C, Schalling D, Sedvall G. A comprehensive psychopathological rating scale. Acta Psychiatr Scand Suppl 1978; 271: 5-27.

19 Montgomery S, Åsberg M, Jörnestedt L, Thoren P, Träskman L, McAuley R, et al. Reliability of the CPRS between the disciplines of psychiatry, general practice, nursing and psychology in depressed patients. Acta Psychiatr Scand Suppl 1978; 57: 29-32.

20 Brus O, Nordanskog P, Bave U, Cao Y, Hammar A, Landen M, et al. Subjective memory immediately following electroconvulsive therapy. J ECT 2017; 33: 96-103.

21 Guy W. Clinical Global Impressions. In ECDEU Assessment Manual for Psychopharmacology: Revised 1976: 217-21. National Institute of Mental Health, 1976

22 Van Buuren S. Flexible Imputation of Missing Data. Chapman \& Hall/CRC, 2018.

23 Haubo R, Christensen B. A Tutorial on Fitting Cumulative Link Mixed Models with clmm2 from the ordinal Package. R Package Documentation (rdrr.io), 2019.

24 Squire LR, Slater PC. Electroconvulsive therapy and complaints of memory dysfunction: a prospective three-year follow-up study. Br J Psychiatry 1983; 142: $1-8$.

25 Freeman C, Kendell R. ECT: I. Patients' experiences and attitudes. $\mathrm{Br} \mathrm{J}$ Psychiatry 1980; 137: 8-16.

26 Petersen JZ, Porter RJ, Miskowiak KW. Clinical characteristics associated with the discrepancy between subjective and objective cognitive impairment in depression. J Affect Disord 2019; 246: 763-74.

27 Semkovska M, Landau S, Dunne R, Kolshus E, Kavanagh A, Jelovac A, et al. Bitemporal versus high-dose unilateral twice-weekly electroconvulsive therapy for depression (EFFECT-Dep): a pragmatic, randomized, non-inferiority trial. Am J Psychiatry 2016; 173: 408-17.
OPEN

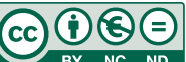

